\title{
Terapia Tópica com Pimecrolimus em Lesão Cutânea Refratária de Lúpus Eritematoso Sistêmico
}

\section{Topical Therapy with Pimecrolimus in Refractory Cutaneous Lesion of Systemic Lupus Erythematosus}

\author{
Leandro Vasconcellos Rangel ${ }^{(1)}$, Juliana de Miranda Santiago ${ }^{(2)}$, Júlia Constança Conceição Souza ${ }^{(2)}$, Larissa \\ Fraga Lima Nascimento ${ }^{(2)}$, Mittermayer Barreto Santiago ${ }^{(3)}$
}

\section{RESUMO}

O rash do lúpus eritematoso sistêmico (LES) é usualmente tratado com corticosteróides tópicos, porém seu uso prolongado causa efeitos adversos cutâneos. Pimecrolimus é um protótipo da classe de agentes imunosupressores tópicos com grande potencial para o tratamento de doenças inflamatórias cutâneas. Terapia tópica com pimecrolimus foi aplicada em lesões cutâneas de face em um caso de LES refratária a esteróides sistêmico e tópico. Houve uma marcante regressão das lesões de pele, após terapia com pimecrolimus. Este tratamento foi bem tolerado, sem efeitos colaterais nesta paciente. Pimecrolimus tópico parece ser uma nova ferramenta para o manejo de lesões cutâneas refratárias em LES.

Palavras-chave: pimecrolimus, lúpus eritematoso sistêmico, doença inflamatória cutânea.

\section{INTRODUÇÃO}

Lúpus eritematoso sistêmico (LES) é uma doença auto-imune sistêmica caracterizada pela presença de autoanticorpos, principalmente, contra componentes do núcleo celular, afetando diversos órgãos como pele, articulações, rins e vasos. Múltiplas disfunções na regulação do sistema imunológico estão envolvidas na sua patogênese, destacando-se a hiper-reatividade de linfócitos $\mathrm{T}$ a antígenos próprios. Auto-anticorpos dependentes de linfócitos $\mathrm{T}$ são formados e geram complexos imunes com antígenos próprios, que se depositam em diversos tecidos levando a lesões teciduais, inclusive cutâneas ${ }^{(1)}$.

\begin{abstract}
The rash of systemic lupus erythematosus (SLE) is usually treated with topical corticosteroids, but prolonged use causes adverse cutaneous side-effects. Pimecrolimus is a prototype of a class of topical immunosuppressive agents with great potential for the treatment of inflammatory skin diseases. Topical pimecrolimus therapy was applied to facial skin lesions in one case of SLE refractory to topical and systemic steroids. There was a marked regression of the skin lesions after pimecrolimus therapy. This treatment was well tolerated without side effects in this patient. Topical pimecrolimus seems to be a new tool for the management of refractory skin lesions in lupus erythematosus.
\end{abstract}

Keywords: pimecrolimus, systemic lupus erythematosus, inflammatory skin disease.

Até $80 \%$ dos pacientes com LES evoluem com lesões cutâneas, mais comumente em áreas expostas à radiação $\operatorname{solar}^{(2)}$. Estas lesões podem se apresentar de formas variadas como rash malar, lesões discóides, maculopapulares ou bolhosas. Apesar do acometimento cutâneo normalmente não levar a risco de vida, estas lesões podem trazer conseqüências ao status emocional dos pacientes, em sua grande maioria mulheres jovens, por afetar desfavoravelmente a aparência de forma importante em muitos casos. Opções de tratamento para estas situações incluem corticosteróides sistêmicos ou tópicos, agentes antimaláricos, azatioprina, dapsona, talidomida ${ }^{(3-5)}$. No entanto, algumas vezes a 
resposta terapêutica a estes agentes são insatisfatórias ou o risco de efeitos adversos não são aceitáveis para doença restrita à pele ${ }^{(3,4,6)}$.

Alternativas terapêuticas tópicas com baixo risco de efeitos colaterais vêm sendo empregadas nos últimos anos em lesões cutâneas do LES. Na classe dos macrolídeos imunosupressores que atuam inibindo o sistema calcineurina, duas drogas vêm sendo empregadas em formulações tópicas: tacrolimus e, mais recentemente, o pimecrolimus ${ }^{(1-12)}$. Enquanto tacrolimus e a ciclosporina A, outra droga pertencente a esta classe, foram originalmente desenvolvidas para prevenção de rejeição a órgãos em pacientes transplantados, o pimecrolimus a $1 \%$ na forma de creme foi desenvolvido especificamente para o tratamento de doenças inflamatórias cutâneas. Pimecrolimus tópico vem sendo empregado com eficácia e segurança em dermatite atópica ${ }^{(13)}$.

Existem poucos trabalhos descrevendo o emprego de pimecrolimus em lúpus eritematoso cutâneo (LEC). Nós descrevemos um caso de uma paciente com LES e acometimento cutâneo na forma de rash malar resistente a outras terapias que teve uma resposta significativa com o uso de pimecrolimus, além de realizarmos uma revisão de literatura sobre o emprego da droga em LEC.

\section{DESCRIÇÃO DO CASO}

MCCR, 38 anos, sexo feminino, há cerca de dois anos começou a cursar com quadro de poliartrite associada à alopecia. Realizou FAN (HEp-2) que foi reagente 1/5120, padrão homogêneo. Teve, então, diagnóstico de LES. Há cerca de nove meses procurou o nosso Serviço com quadro de febre de etiologia não definida (duração de 30 dias), rash cutâneo em face e colo, artrite, leucopenia, plaquetopenia e vasculite em polpas digitais, necessitando de internamento.

Após 26 dias de internamento, cursou com melhora do quadro, hematológico, vasculítico e febril, porém mantendo rash cutâneo em face em forma de asa de borboleta, a despeito do uso de prednisona $60 \mathrm{mg} /$ dia e azatioprina $100 \mathrm{mg} /$ dia. A prescrição da alta hospitalar foi a seguinte: prednisona $60 \mathrm{mg} /$ dia, carbonato de cálcio $1.000 \mathrm{mg} /$ dia, fluoxetina $20 \mathrm{mg} /$ dia e, diante da intensa atividade cutânea a despeito de altas doses de corticóide e uso de imunossupressor, foi iniciado pimecrolimus creme a $1 \%$, uso tópico em face e colo. É importante salientar que a avaliação oftalmológica evidenciou presença de retinose pigmentar, o que nos levou a evitar o uso de cloroquina e derivados. A idade da paciente e a dificuldade de monitoramento adequado da anticoncepção contra-indicavam o emprego de talidomida.

A paciente cursou com melhora significativa do rash cutâneo facial e da região do colo após quatro semanas de uso (Figura 1) e manteve melhora mesmo com a redução progressiva da prednisona até $5 \mathrm{mg} /$ dia. Por dificuldade temporária de aquisição do pimecrolimus, a paciente deixou de usar a substância por algumas semanas, com recidiva do quadro, o que foi revertido com a reintrodução da mesma. Durante a terapia com o pimecrolimus tópico, a paciente não apresentou qualquer tipo de efeito adverso local ou sistêmico até o follow-up de 20 semanas.

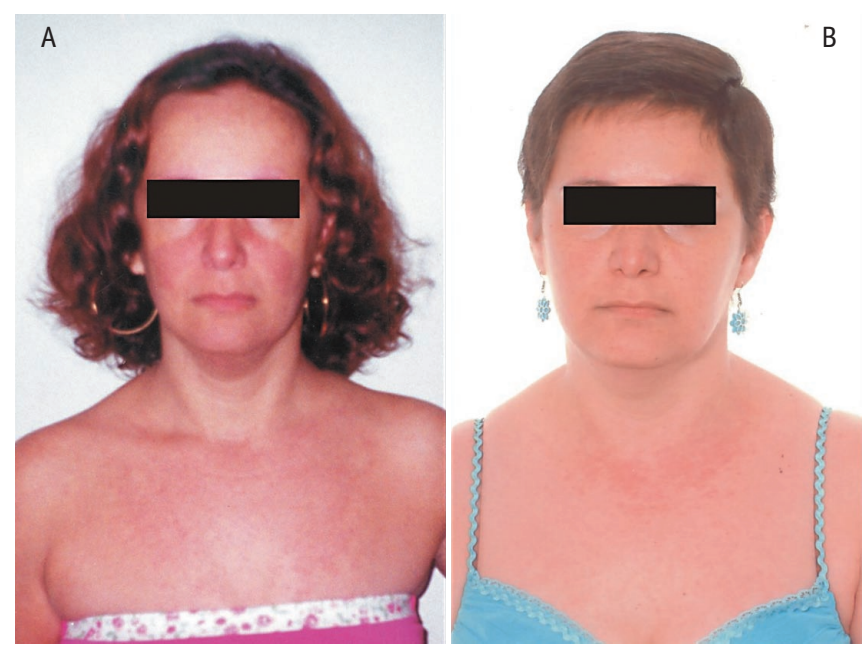

Figura 1 - Lúpus eritematoso sistêmico com exuberante rash cutâneo, antes (A) e depois (B) do uso de pimecrolimus tópico.

\section{DISCUSSÃO}

O LES é uma doença auto-imune, envolvendo órgãos e sistemas, que apresenta uma grande variedade de manifestações clínicas. As lesões de pele são encontradas na maioria dos pacientes.

A principal razão para o desenvolvimento de drogas alternativas aos corticosteróides tópicos são os efeitos colaterais destes. Os imunossupressores macrolactâmicos, como o tacrolimus e, recentemente, o pimecrolimus, representam uma nova classe de drogas que podem superar o uso dos corticosteróides no tratamento local de lesões crônicas inflamatórias de pele ${ }^{(2)}$. Outros benefícios que estas drogas podem trazer é a possibilidade de evitar o uso de medicações com potencial de efeitos sistêmicos indesejáveis nos quadros restritos à pele, além de permitir uma alternativa terapêutica aos pacientes com lesões refratárias aos tratamentos disponíveis. Recentes estudos têm demonstrado que o uso do pimecrolimus a $1 \%$ tópico não está envolvido 
com o aparecimento de telangiectasias e atrofia de pele, comumente observados com o uso dos corticóides ${ }^{(2,4,13)}$.

A ativação de células T cutâneas está envolvida na patogênese do lúpus eritematoso discóide (LED). O cálcio intracelular aumenta com a ativação das células $\mathrm{T}$ e liga-se à calmodulina, a qual ativa o sistema calcineurina, uma fosfatase ativada pelo cálcio. Esta promove a defosforilação de fatores nucleares de ativação de células $\mathrm{T}$, que atuam em regiões do DNA da célula causando o estímulo à produção de citocinas de perfil inflamatório. O pimecrolimus atua inibindo a produção e liberação de citocinas pró-inflamatórias, como interleucina 2 (IL-2), interferon- $\gamma$, IL-4, IL8 , IL-10 e o TNF- $\alpha^{(4)}$, pela sua ação inibitória no sistema calcineurina. Este também age seletivamente em linfócitos T e mastócitos, diferentemente dos corticosteróides e tacrolimus que são menos seletivos, sendo estas células cruciais na patogênese de muitas doenças cutâneas inflamatórias, incluindo as lesões cutâneas do LES. Em contraste com os corticosteróides, pimecrolimus e tacrolimus não afetam células endoteliais e fibroblastos cutâneos, desta forma não induzem telangiectasias e atrofia cutânea ${ }^{(13)}$.

$\mathrm{O}$ alto peso molecular do pimecrolimus (aproximadamente 800 Daltons) em relação aos corticosteróides tópicos (aproximadamente 470 Daltons) contribui para dificultar o alcance da droga na circulação sistêmica e promover efeitos colaterais.

Existem alguns estudos que demonstram a eficácia do tacrolimus tópico em diversas formas do $\operatorname{LEC}^{(1,3,7}$, $\left.{ }^{10}, 11\right)$, incluindo $\operatorname{LED}^{(5,8,9)}$, porém informações sobre $\mathrm{O}$ pimecrolimus ainda são escassas ${ }^{(2,4,5,12,13)}$. Dados da literatura sugerem que o tratamento tópico com pimecrolimus em lesões de pele do LES também é bastante efetivo e seguro. Zabawski ${ }^{(12)}$ foi o primeiro a relatar um caso demonstrando a regressão das lesões de pele do LED com o uso do pimecrolimus. Kreuter et al ${ }^{(2)}$ observaram melhora significativa das lesões de pele, com o uso dessa mesma droga, duas vezes ao dia, em 11 pacientes com diferentes formas de LEC. Mais recentemente, Tlacuilo-Parra et $\mathrm{al}^{(4)}$ observaram resultados semelhantes em estudo com dez pacientes. Estes observaram uma melhor resposta ao uso do pimecrolimus tópico nas lesões de lúpus eritematoso cutâneo subagudo (LECS) e lúpus eritematoso tumidus
(LET) quando comparados às lesões discóides mais antigas. Este fato seria devido à presença de hiperceratose mais intensa nestas lesões dificultando a penetração transdérmica da droga. Estudos com tacrolimus tópico também sugerem esta menor resposta quando empregados em lesões discóides ${ }^{(10)}$. No entanto, o emprego de oclusão com adesivos de hidrocolóides pode melhorar a penetração e a eficácia da droga em lesões hiperceratóticas ${ }^{(2)}$.

Respaldados em tais experiências, optou-se pelo uso do pimecrolimus em nossa paciente que apresentava rash cutâneo malar resistente ao uso de altas doses de prednisona $(60 \mathrm{mg} /$ dia $)$ associado à azatioprina $100 \mathrm{mg} /$ dia. Uma vez que apresentava retinose pigmentar evitou-se o uso de cloroquina e derivados. Visto que a terapia sistêmica oral levou ao controle da atividade de doença em diversos sistemas, exceto da atividade cutânea, creditou-se ao uso do pimecrolimus tópico como fator fundamental para controle da atividade inflamatória cutânea. Adicionalmente, a suspensão temporária da medicação tópica, mesmo mantendo a prednisona e a azatioprina, levou à reativação da lesão cutânea o que foi revertida com a reintrodução do pimecrolimus. Observou-se também que a resposta terapêutica foi relativamente rápida, cerca de 4 semanas, e manteve-se por até a última reavaliação pós 20 semanas de terapia, mesmo diante da redução importante da dose da prednisona. Destaca-se também a segurança da droga, vista a ausência de qualquer efeito adverso do emprego do pimecrolimus no presente caso, sendo que trabalhos anteriores confirmam a ausência de efeitos sistêmicos até o momento descritos, e relatam efeitos locais como sensação de queimor, rubor, prurido e foliculites ${ }^{(4)}$ que tendem a progressivamente reduzir de intensidade com uso regular.

Baseando-se no presente caso e em dados da literatura, conclui-se que o pimecrolimus parece ser uma alternativa segura e efetiva no tratamento de lesões cutâneas do LES, principalmente em lesões resistentes à terapia padrão. Por outro lado, o alto custo da medicação deve ser levado em consideração, pois dificulta seu emprego mais disseminado ficando reservado a casos selecionados. Além disso, os dados são iniciais e ainda são necessários estudos duplo-cegos, placebo-controlados para confirmar esses achados. 


\section{REFERÊNCIAS}

1. Kanekura T, Yoshii N, Terasaki K, Miyoshi H, Kanzaki T: Efficacy of topical tacrolimus for treating the malar rash of systemic lupus erythematosus. Br J Dermatol 148: 353-6, 2003.

2. Kreuter A, Gambichler T, Breuckmann F et al: Pimecrolimus $1 \%$ cream for cutaneous lupus erythematosus. J Am Acad Dermatol. 51: 407-10, 2004.

3. Lampropoulos CE, Sangle S, Harrison P, Hughes GR, D'Cruz DP. Topical tacrolimus therapy of resistant cutaneous lesions in lupus erythematosus: a possible alternative. Rheumatology (Oxford) 43: 1383-5, 2004.

4. Tlacuilo-Parra A, Guevara-Gutierrez E, Gutierrez-Murillo F et al: Pimecrolimus $1 \%$ cream for the treatment of discoid lupus erythematosus. Rheumatology (Oxford), 1564-8, 2005.

5. de la Rosa Carrillo D, Christensen OB: Treatment of chronic discoid lupus erythematosus with topical tacrolimus. Acta Derm Venereol 84: 233-4, 2004.

6. Walker SL, Kirby B, Chalmers RJ. The effect of topical tacrolimus on severe recalcitrant chronic discoid lupus erythematosus. $\mathrm{Br} \mathrm{J}$ Dermatol 147: 405-6, 2002.
7. Druke A, Gambichler T, Altmeyer P, Freitag M, Kreuter A: 0.1\% Tacrolimus ointment in a patient with subacute cutaneous lupus erythematosus. J Dermatolog Treat 15: 63-4, 2004.

8. Heffernan MP, Nelson MM, Smith DI, Chung JH: $0.1 \%$ tacrolimus ointment in the treatment of discoid lupus erythematosus. Arch Dermatol 141: 1170-1, 2005.

9. Chambers CA: Topical tacrolimus for cutaneous lupus erythematosus. Br J Dermatol 148: 829, 2003.

10. Yoshimasu T, Ohtani T, Sakamoto T, Oshima A, Furukawa F: Topical FK506 (tacrolimus) therapy for facial erythematous lesions of cutaneous lupus erythematosus and dermatomyositis. Eur J Dermatol 12: 50-2, 2002.

11. Bohm M, Gaubitz M, Luger TA, Metze D, Bonsmann G: Topical tacrolimus as a therapeutic adjunct in patients with cutaneous lupus erythematosus. A report of three cases. Dermatology 207: 381-5, 2003.

12. Zabawski E. Treatment of cutaneous lupus with Elidel. Dermatol Online J 8: 25, 2002.

13. Gisondi P, Ellis CN, Girolomoni G: Pimecrolimus in dermatology: atopic dermatitis and beyond. Int J Clin Pract 59: 969-74, 2005. 